Bol. Soc. Bot. México 50:157-159 (1990)

\title{
Nota sobre la propagación y pérdida de viabilidad de las semillas de Chiranthodendron pentadactylon Larr. (Sterculiaceae)
}

El Macpalxóchitl (Chiranthodendron pentadactylon Larr.: Sterculiaceae), emblema de la Sociedad Botánica de México, ha sido objeto de muy pocos estudios. Toledo (1975) estudió el mecanismo de polinización y Estrada (1987) observó algunas de las características de frutos y semillas. A pesar de ser una especie que en época de floración es muy atractiva y de ser muy apreciada por las propiedades medicinales que se le atribuyen, hasta el momento no se conoce cómo propagarla (aunque se considera que es posible multiplicarla vegetativamente por acodos aéreos, E. Linares, com. pers.). Los objetivos del presente trabajo fueron: a) probar si las semillas de Chiranthodendron pentadactylon podían germinar en dos sustratos diferentes (suelo y vermiculita); y b) si las semillas mantenían su viabilidad bajo dos condiciones de almacenamiento (temperatura ambiente y en refrigeración).

Las semillas para la prueba se obtuvieron de 20 frutos, cerrados y sin daño aparente, colectados de cinco árboles en la localidad de Rancho Nuevo, ubicada a una altitud de 2300 msnm, y a 11 km al SE de la Cd. de San Cristóbal de las Casas, Chiapas. Durante la colecta, se observó que la población de Macpalxóchitl de ese sitio estaba compuesta mayoritariamente por individuos adultos (reproductivos), encontrándose pocos jóvenes (no reproductivos) dispersos en el bosque. Dos semanas después de la recolecta, los frutos fueron abiertos manualmente, contándose las semillas presentes en cada uno de ellos. Siendo las semillas del Macpalxóchitl pequeñas y con testa algo dura, se consideró conveniente mantener las semillas de la prueba de almacenamiento bajo condiciones secas y frías, que son las condiciones comúnmente utilizadas para almacenar semillas "ortodoxas" (Vázquez-Yanes, 1987). La prueba de germinación en función del sustrato consistió en sembrar a un centímetro de profundidad, en charolas de germinación, 100 semillas, divididas en diez lotes de 10 semillas cada uno. Los tratamientos fueron: a) cinco lotes en una mezcla de suelo comúnmente utilizada en jardinería (50\% compost, $50 \%$ franco-arcílloso), y b) cinco en vermiculita estéril, como testigo (se dividió en lotes para un mejor control, aunque en el análisis de los resultados fueron agrupados los datos). Para la prueba de almacenamiento se formaron otros 10 lotes de 50 semillas cada uno. Cinco lotes se pusieron a temperatura ambiente (fluctuando alrededor de $\operatorname{los} 15^{\circ} \mathrm{C}$ en la época del año que se hizo la recolecta) y cinco en refrigeración a $4^{\circ} \mathrm{C}$. El objetivo específico era probar en qué condiciones de almacenamiento conservaban mejor la viabilidad. De éstas últimas semillas, un lote de cada tratamiento de almacenamiento, fue sembrado mensualmente en vermiculita estéril, para observar la respuesta de la

García-Franco JG, Perales-Rivera H. 1990. Nota sobre la propagación y pérdida de viabilidad de las semillas de Chiranthodendron pentadactylon Larr. (Sterculiaceae). Boletín de la Sociedad Botánica de México 50: 157-159. 
germinación a través del tiempo de almacenamiento. Las charolas de germinación se mantuvieron en un invernadero proporcionando riego constante (cada 2 ó 3 días) durante el tiempo que duró el trabajo. El conteo de germinación se realizó semanalmente, hasta que ya no se produjeron nuevas plántulas.

Los frutos recolectados tuvieron un promedio de 67 semillas $(s=16.9, n=20)$. Este número es superior al encontrado por Estrada (1987), quien en 125 frutos recolectados en Miahuatlán, Oaxaca, encontró un promedio de 27 semillas por fruto, con una variación de 9 a 64. Aunque el número de semillas por frutos en una especie puede ser muy variable, y está sujeto a la intervención de muchos factores, la diferencia encontrada sugiere la necesidad de realizar estudios poblacionales detallados sobre la variabilidad intraespecífica de $C h$. pentadactylon en función de las condiciones ambientales de los sitios donde crece.

La germinación en los dos sustratos se inició en la cuarta semana, continuando hasta la $11^{\text {a }}$ semana. Se obtuvo el $74 \%$ de germinación en el tratamiento de vermiculita, y sólo el $39 \%$ en las semillas que fueron sembradas en el suelo. Hay que hacer notar, que aunque la germinación final de los dos tratamientos no fué significativamente diferente $\left(\mathrm{x}^{2}=10.84, \mathrm{P}<0.05\right)$, en la vermiculita se obtuvo casi el doble de la germinación observada en el suelo.

De las semillas almacenadas a temperatura ambiente emergieron coleópteros, Acanthoscelides guatemala Johnson (Bruchidae), destruyendo el 11\% de las semillas. Estrada (1987) encontró en las semillas del Macpalxóchitl individuos de Acanthoscelides obtactus (Coleoptera: Bruchidae) y de Callosobructus sp. (Coleoptera:Anobiidae). Lo anterior sugiere que las semillas de Chiranthodendron pentadactylon están asociadas con las especies de coleópteros que habiten en los diferentes sitios donde esta planta se distribuye. Se ha probado que la depredación de semillas por insectos limita la abundancia y reclutamiento de las poblaciones de arbustos en climas templados (Louda, 1982), pudiendo ser un factor importante en su dinámica, si el suplemento de semillas es bajo y limita sustancialmente el reclutamiento de plántulas (Harper, 1977). Aunque la depredación de las semillas del Macpalxóchitl no sea extrema, puede ocasionar una reducción en el potencial de regeneración de la especie.

La pérdida de viabilidad en las semillas almacenadas fué notable. Al primer mes, las semillas presentaron $10 \%$ y $12 \%$ de germinación, para el tratamiento frío y a temperatura ambiente, respectivamente. A los dos meses de almacenamiento, únicamente se logró una germinación de $2 \%$ a $4^{0} \mathrm{C}$ y $4 \%$ a temperatura ambiente. En las siembras posteriores no germinó ninguna semilla. Lo anterior nos sugiere que las semillas pierden rápidamente su viabilidad, y que las condiciones de almacenamiento utilizadas no resultaron ser adecuadas. Esto nos permite suponer que las semillas en los bosques también reduzcan considerablemente su viabilidad con el transcurso del tiempo.

Los resultados obtenidos sugieren que la propagación de Ch. pentadactylon por semilla puede realizarse con relativa facilidad, únicamente teniendo en cuenta la rapidez 
con que pierden la viabilidad las semillas. Lo anterior abre un campo de investigación fisiológica sobre las condiciones en que deben almacenarce para obtener una adecuada germinación. Por otra parte, el porcentaje de germinación obtenido en el suelo, la destrucción de parte de sus semillas por escarabajos y la pérdida de la viabilidad (aunado a la rápida modificación de los ecosistemas y al pastoreo en los bosques, principalmente por ganado caprino que se presenta en casi toda la parte alta del estado de Chiapas), podrían ser las causas que provocan reducción en la regeneración natural. Consideramos que estos hechos sean los causantes de que se haya observado en el sitio de recolecta mayor proporción de individuos adultos que jóvenes. Por último, la evidente falta de conocimiento sobre Chiranthodendron pentadactylon, hace imprescindible realizar estudios a varios niveles (ecológicos, fisiológicos, etc.), que nos permitan obtener un mayor y mejor conocimiento de esta especie.

Agradecimientos. Agradecemos a dos revisores anónimos las sugerencias realizadas a las versiónes anteriores del presente manuscrito. A Victor Rico-Gray los comentarios y observaciones al escrito final, y a Arturo Bonet su colaboración para lograr la iđentificación del escarabajo. Y al INIREB las facilidades otorgadas para el uso de sus instalaciones durante el estudio.

\section{LITERATURA CITADA}

ESTRADA, L.E. 1987. El fruto de Macpalxochicuahuitl (Chiranthodendron pentadactylon Larr.): Tamaño, semillas, plagas y polinización manual. En: Resúmenes del X Congreso Mexicano de Botánica. Sociedad Botánica de México.

HARPER, J.L. 1977.Population biology of plants. Academic Press, London.

LOUDA, S.M. 1982. Limitation of the recruitment of the shrub Haplopappus squarrosus (Asteraceae) by flower- and seed-feeding insects. J. Ecol. 70:43-53.

TOLEDO, V.M. 1975. Chiranthodendron pentadactylon Larreategui (Sterculiaceae): una especie polinizada por aves percheras. Bol. Soc. Bot. México 35:59-67.

VÁZQUEZ-YANES, C. 1987. Los bancos de almacenamiento de semillas en la conservación de especies vegetales. Ciencia 38:239-246.

JOSÉ GUADALUPE GARCÍA-FRANCO, Instituto de Ecología, A.C. Apdo. Pcst. 63, 91000 Xalapa, Ver. México y HUGO R. PERALES RIVERA, Centro de Botánica, Colegio de Postgraduados, Montecillos, México. 